\title{
A existência de uma cultura escolar de reprovação no ensino de Cálculo
}

\author{
Maria Cristina Araújo de Oliveira \\ Professora, UFJF, Juiz de Fora/MG \\ mcrisoliveira6@gmail.com
}

\author{
Marcos Ribeiro Raad \\ Professor, Colégio Academia de Comércio e do \\ Centro de Ensino Superior, Juiz de Fora/MG \\ marcosraad@ibest.com.br
}

\begin{abstract}
Resumo
A partir de uma pesquisa de cunho histórico sobre o ensino de Cálculo Diferencial e Integral, o presente artigo $^{1}$ identifica a reprovação como um elemento da cultura de ensino dessa disciplina. Estudos históricos sobre a disciplina e o seu ensino desde os anos 50 até os 80 do século passado, permitem avaliar que, apesar da didática empregada pelo professor; do uso de bons livros; dos esforços dos Departamentos de Matemática das instituições de ensino superior em ofertar cursos intensivos, em aumentar o número de aulas, em estabelecer cursos de Matemática Básica, em redirecionar a disciplina de seu aspecto teórico-formalista para uma abordagem mais aplicada; a reprovação se constituiu num problema crônico, uma verdadeira tradição. Contudo a perspectiva histórica também permite avaliar que a mudança desse cenário se relaciona com a renovação o corpo docente e a crescente participação de educadores matemáticos nos cursos de formação de professores.
\end{abstract}

Palavras-chave: História da educação matemática; história das disciplinas; cultura do ensino de Cálculo; ensino de Cálculo; reprovação em Cálculo.

\section{The existence of a school culture of failure in the teaching of Calculus}

\begin{abstract}
Based upon historical studies on the teaching of the subject Differential and Integral Calculus, this article perceives that course failure is used as a culture of teaching. From historical studies on the teaching of the subject since the 50 s until the 80 s of last century, the study points to the conclusion that, despite the teaching employed by the teacher, the use of good books, the efforts of the Departments of Mathematics of the higher education institutions in offering intensive courses, to increase the number of classes to establish basic mathematics courses, to redirect the course of his formalist-theoretical aspect to a more applied, the course failure is a chronic problem, a tradition. On the other hand, the historical studies also give us the feeling that a change in the situation must be related to a renovation of the teachers and a growing participation of the mathematics educators in the courses of formation of the new teachers.
\end{abstract}

Keywords: History of mathematics education; history of the disciplines; culture of the teaching of Calculus; teaching Calculus; Calculus reproach.

1. Deixamos registrado nosso agradecimento ao Professor Wagner Rodrigues Valente pelas sugestões para a elaboração desse artigo. 


\section{O Cálculo Diferencial e Integral: os problemas de seu ensino e aprendizagem}

Os processos de ensino e de aprendizagem de Cálculo são fontes inesgotáveis de pesquisas em várias partes do mundo. Diversos estudos mostram as dificuldades de aprendizagem nesta disciplina; e ainda que divirjam quanto à natureza destes obstáculos, se de ordem didática ou epistemológica, em alguma medida, vinculam e justificam tais dificuldades à alta reprovação na disciplina.

Ao investigar as dificuldades que emergem do ensino de Cálculo, Barufi (1999), tomando como referencial teórico a rede de conhecimentos e significados e entendendo que o livro didático escolhido pelo professor constitui um forte indício do tratamento que será dado ao curso, lançou mão da análise de 24 livros de Cálculo Diferencial e Integral publicados entre os anos de 1945 e 1997 para discutir esta problemática. A análise dos livros mostrou que tais dificuldades não residem na falta de bons livros. Barbosa (2004) atribui o insucesso na aprendizagem de Cálculo Diferencial e Integral a um sistema didático constituído por práticas de ensino e de estudo tradicionais.

Tratando especificamente do Teorema Fundamental do Cálculo (TFC), Anacleto (2007) indica que as dificuldades apresentadas pelos alunos no entendimento desse teorema decorrem da falta de articulação entre as noções de derivada, integral e continuidade.

Ainda sobre o TFC, Escarlate (2008) utilizou como referencial teórico as noções de imagem de conceito e definição de conceito (TALL \& VINNER, 1981) para mostrar que grande parte dos alunos adquire uma ideia imprecisa da definição de integral definida, e que essa concepção provoca erros até mesmo em situações consideravelmente simples.

Numa perspectiva propositiva, Cury (2007) avalia que é possível compreender como ocorre o processo de construção de conhecimento dos alunos por meio de suas produções escritas. Serão estas produções que nortearão as estratégias a serem delineadas para a superação das dificuldades apresentadas pelos alunos relativamente à aprendizagem de Cálculo.

Os trabalhos aqui apresentados tratam a questão da dificuldade de aprendizagem em Cálculo, e a consequente reprovação nessa disciplina, em duas perspectivas: uma de identificação de razões para as dificuldades e outra na proposição de alternativas para a superação das mesmas. Nenhum desses trabalhos, assim como acontece com tantos outros existentes embora não citados aqui, discute a reprovação como um elemento do contexto onde ocorre esse ensino. Mais precisamente, não há, ao que tudo indica, estudos que considerem os condicionantes das práticas pedagógicas dos professores que ensinam a disciplina.

Este estudo intenta analisar os problemas relativos ao ensino e à aprendizagem do Cálculo Diferencial e Integral sob a perspectiva de que qualquer prática pedagógica é condicionada por elementos que vão além daqueles que dizem respeito às iniciativas 
próprias de cada professor. E esses elementos, como se procurará mostrar, têm caráter determinante no modo como são exercidas as práticas pedagógicas. Tal perspectiva advoga a existência de uma cultura escolar. Colocado de outro modo, o objetivo deste estudo é o de identificar os elementos componentes de uma ambiência de reprovação em Cálculo Diferencial e Integral. A existência dessa cultura será tratada neste artigo sob um recorte temporal que compreende desde a década de 1950 até a década de 1980 .

\section{Alguns elementos teórico-metodológicos}

O desenvolvimento do conceito de cultura escolar pode ser creditado à bases teórico-metodológicas ligadas à chamada História Cultural. Dada a sua complexidade e extensão, não é tarefa simples caracterizar em poucas linhas, como se dá a pesquisa que tem por referência os estudos histórico-culturais. No entanto, algumas balizas parecem indicar o caminho a seguir na adoção dessa perspectiva e podem caracterizar, mesmo que de forma breve, essa via de estudos. Uma delas refere-se ao tratamento dado ao termo cultura. O historiador francês Roger Chartier menciona haver duas famílias de significados para ele: uma delas é a que designa por cultura as obras e os gestos que, em uma dada sociedade se subtraem das urgências do cotidiano para submeterem-se a um juízo estético ou intelectual; a outra, se refere às práticas ordinárias através das quais uma sociedade ou um indivíduo vivem e refletem sobre sua relação com o mundo, com os outros ou consigo mesmo (CHARTIER, 2007, p. 50). Assim, será considerando esta segunda acepção, que toma a noção de cultura agarrada a um grupo e à sua vida comum de existência, que nos localizamos no âmbito de uma História Cultural; em específico, à vida cotidiana das escolas, das universidades, aos significados construídos no meio escolar ou acadêmico que dão vida e funcionamento ao dia-a-dia pedagógico. Ou seja, ao que se pode chamar de cultura escolar. E, nesse contexto, interessam os modos de tratar os diferentes saberes presentes na formação do professor de matemática, expressos através de suas disciplinas.

Vinão Frago (2008, p.199) aponta algumas diretrizes para quem pretende trabalhar na construção da história de uma disciplina acadêmica: conhecer seu lugar, sua presença, suas denominações, os objetivos explícitos e implícitos, os discursos que a legitimam como disciplina acadêmica, seus conteúdos, a formação dos professores desta disciplina, as fontes (livros-texto, diários, cadernos, memórias, atas de reuniões, documentos particulares).

No âmbito da História Cultural, o historiador Dominique Julia define o conceito de cultura escolar como: "um conjunto de normas que definem conhecimentos a ensinar e inculcar, e um conjunto de práticas que definem a transmissão desses conhecimentos e a incorporação desses comportamentos" (JULIA, 2001, p. 10). 
Assim, mobilizando o conceito de cultura escolar, ou seja, investigando normas e práticas da disciplina ao longo do tempo na afirmação da disciplina Cálculo Diferencial e Integral como elemento do currículo de formação do professor de matemática, busca-se resposta à questão considerada crônica para os baixos índices de aprovação na disciplina. A prática docente permite observar o temor que a disciplina Cálculo suscita nos estudantes. Mesmo que o curso aborde apenas conteúdos básicos de Matemática e trate somente as ideias do Cálculo, é, em geral, considerado difícil pelos alunos, especialmente no que se refere à aprovação. Esse código entre os estudantes contribui para o estabelecimento de um mito de que é normal a reprovação nessa disciplina. Entre os professores também se nota certa naturalidade quanto aos altos índices de reprovação.

Essa ideia cristalizada sobre a disciplina de Cálculo como uma barreira a ser vencida é transmitida de geração em geração pela cultura acadêmica. Nas palavras de Chervel (1990):

Fruto de um diálogo secular entre os mestres e os alunos, as disciplinas escolares constituem por assim dizer o código que duas gerações, lentamente, minuciosamente, elaboraram em conjunto para permitir a uma delas transmitir à outra uma cultura determinada. (CHERVEL, 1990, p.222).

Como se pode verificar pela investigação histórica à respeito do ensino de disciplinas acadêmicas ${ }^{2}$, a transmissão cultural analisada por Chervel não é exclusividade da escola, fenômeno similar ocorre no ensino superior, no ambiente acadêmico.

A possibilidade de problematizar as disciplinas acadêmicas de modo a admiti-las como objeto de investigação histórica se verifica pelo desenvolvimento de pesquisas que demonstram a necessária e inevitável criação de disciplinas a partir dos saberes de referência, do público a quem o ensino se dirige, do contexto socioeconômico e político da instituição de ensino, da cultura da instituição, entre tantos outros fatores.

No ofício de historiador da educação matemática o objetivo deste artigo é desnaturalizar a problemática da reprovação em Cálculo e identifica-la como um elemento da cultura do ensino dessa disciplina (VALENTE, 2007).

\section{O ensino de Cálculo nas décadas de 1950 e 1960}

O ambiente no curso de Matemática da Faculdade de Filosofia Ciências e Letras da (FFCL) da USP, na década de 1950, era regido pelos chamados cursos magistrais. 
Tal denominação foi cunhada no projeto de pesquisa LE COURS MAGISTRAL: MODALITÉS ET USAGES (XVI ${ }^{e}$-XX $X^{e}$ siècles) Pratiques orales et pratiques écrites dans la constitution et la diffusion des savoirs, coordenado pela professora Annie Bruter pesquisadora do Instituto Nacional de Pesquisa Pedagógica da França, a partir de 2005.

Os cursos magistrais são constituídos por associações da produção e da transmissão do saber por meio de práticas orais e escritas.

Durante as aulas de um curso magistral os professores expõem suas ideias e os conceitos que desejam transmitir aos alunos, mas também na interação com seu grupo de alunos essas ideias são ajustadas, afinadas para uma melhor comunicação (OLIVEIRA, 2007, p.62).

Pelo estudo das notas dos participantes pode-se verificar em que medida cursos publicados sobre a forma de manuais, apostilas ou mesmo livros tem sua origem em notas de aulas e o papel dessas na constituição e na difusão de novos saberes.

Oliveira (2007) investigou o curso de Matemática da FFCL da USP realizado pelo Professor Ubiratan D’Ambrosio no período de 1951 a 1954. Um ambiente de curso magistral pode ser percebido no depoimento do Professor sobre a condução da aula como um momento de criação e não somente de reprodução de conhecimento.

Eu não fui aluno do Jean Dieudonné ou do Fantappiè, eles vinham com um estilo de dar aula que era o estilo do Catedrático europeu. Cada vez que entrava em uma aula o professor sentia isso ... essa minha aula é um momento muito importante onde eu estou criando. Este estilo passou para os meus professores, então aprendemos a ser professores com eles. Este estilo passou, e eles davam aula desse jeito também, momento de aula não é momento para você papagaiar simplesmente o que os outros escreveram, a aula tem que ser sua, essa é a responsabilidade do catedrático... (D'AMBROSIO apud OLIVEIRA 2007, p. 61- 62).

Professores como Jacy Monteiro ${ }^{3}$, Omar Catunda ${ }^{4}$, Benedito Castrucci ${ }^{5}$ e Elza Gomide $^{6}$ reproduziam seus mestres, os catedráticos europeus que os influenciaram

3. Professor catedrático de Álgebra da FFCL da USP.

4. Professor catedrático de Análise Matemática da FFCL da USP.

5. Professor catedrático de Geometria da FFCL da USP.

6. Professora do Departamento de Matemática da FFCL da USP durante a segunda metade do século XX, foi professora de D'Ambrosio na graduação. 
em sua formação como Luigi Fantappiè, ${ }^{7}$ Giacomo Albanese ${ }^{8}$, e Jean Dieudonné durante as décadas de 30 e 40 do século passado.

Mas esses cursos magistrais não produziam melhores resultados em termos de aprovação. A reprovação em Cálculo, na época Análise Matemática, era elevada, conforme se pode constatar no relato do Professor Morettin.

O professor Catunda dava aulas de Análise Matemática, um curso muito difícil [...] Interessante que quando ele dava aulas, praticamente não escrevia na lousa, ficava falando, falando, mais parecia um professor de Filosofia do que de Matemática. Lembro que no começo do ano éramos em média 60 alunos, mas no fim passavam uns 5; nas provas, a maioria era reprovada. Era um excelente professor (SILVA, 2000).

Percebe-se no depoimento do Professor Morettin que o desastre do curso em termos de reprovação respaldava ainda mais a qualidade do Professor Catunda. Vê-se assim o processo de construção de uma ideia ainda frequente entre professores de Matemática de que: "curso bom é curso que reprova".

A partir da década de 1960 o ensino de Cálculo na USP sofre profundas transformações, um primeiro fator que contribuiu para esse processo de mudança foi a aposentadoria em 1962 do Professor Catunda, uma referência no ensino de Análise Matemática da época. Como destaca Chervel (1990) um dos fatores importantes no processo de transformação de uma disciplina é a renovação do corpo docente.

Aliada à aposentadoria do referido professor, ocorre também a adoção de livros americanos de Cálculo que propunham, ao enfatizar a parte operacional do Cálculo, na manipulação algébrica de limites, derivadas e integrais, uma abordagem diferente da escola européia no ensino da Análise Matemática.

Em 1964 ocorreu na USP a implementação da disciplina Cálculo propriamente dita, em substituição à Análise Matemática, antiga "responsável” por apresentar aos alunos os conceitos de limites e os campos da derivação e integração. Ainda que tais cursos tivessem um aspecto teórico e formal, bastante diferente das premissas dos cursos atuais de Cálculo, fossem mais próximos dos cursos da década de 1950 no

7. Foi um dos professores estrangeiros contratados para reger a cadeira de Análise Matemática da FFCLUSP, permanecendo durante o período compreendido entre 1934 e 1939 (DUARTE, 2007).

8. Chegou ao Brasil em 1936, tornando-se o segundo matemático italiano, depois de Fantappié, a exercer atividade docente no Departamento de Matemática. Albanese foi assessorado por Benedito Castrucci (DUARTE, 2007).

9. Professor Titular do Departamento de Estatística da USP obteve a graduação em Matemática pela USP em 1962. 
tocante ao rigor ao se utilizarem da linguagem de épsilons e deltas, havia também um caráter de manipulações algébricas em seu ensino.

A Professora Vera Giusti de Souza ${ }^{10}$ que foi aluna do curso de Matemática da USP tendo ingressado em 1966, logo que se formou foi contratada como professora assistente. Em entrevista relatou a sua experiência como aluna do curso e posteriormente como docente do Departamento de Matemática. Sua prática como professora de Cálculo Diferencial e Integral muito diferiu daquela utilizada por seus professores no período em que foi estudante. Segundo a Professora, logo que começou a lecionar foi trabalhar com a Professora Iracema Martins Bund, que tinha uma proposta bastante diferenciada para conduzir a disciplina que não se pautava na exposição de teoremas e demonstrações como Vera havia aprendido. A Professora Iracema, recém-chegada de seu doutorado nos EUA, implementou a metodologia de estudo dirigido com os alunos. As aulas eram preparadas pelo grupo de docentes que trabalhavam com os cursos de Matemática e Física; aos alunos eram propostas leituras de livros em sala de aula, muitos deles americanos, e o desenvolvimento de atividades a partir de um roteiro de questões e exercícios. Estes eram entregues todas as aulas e os professores corrigiam e traziam comentados de uma aula para a outra.

$\mathrm{Na}$ avaliação da Professora Vera, a mudança de metodologia e as próprias adequações feitas aos conteúdos da disciplina Cálculo, menor ênfase nas definições e demonstrações com épsilons e deltas e maior valorização do saber fazer, levaram a uma redução no índice de reprovação nas turmas onde tais transformações foram implementadas, alunos de Física e Matemática. Nesse período o índice de reprovação caiu para cerca de $30 \%$ nessas turmas enquanto que na Engenharia, por exemplo, chegava a $70 \%$.

\section{O ensino de Cálculo nas décadas de 1970 e 1980}

Tomando como referência outro ambiente de ensino, a Universidade Federal de Juiz de Fora ${ }^{11}$, Raad (2012) identificou elementos da cultura de ensino de Cálculo nas décadas de 1970 a 1980, nesta instituição.

Segundo Schubring (2010), estudos de caso podem se constituir em particularidades que revelam diferenças locais ou regionais, mas também podem contribuir para mostrar a permanência ou estabilidade de comportamentos, tendências, propostas.

10. Professora do Instituto de Matemática e Estatística da USP por 27 anos.

11. A Universidade de Juiz de Fora foi criada em 1960 como resultado de promessa do então Presidente Juscelino Kubitschek (Fragoso 2011, p. 52), passou a ser denominada Universidade Federal de Juiz de Fora em 1965. 
As notas de aula de Cálculo Diferencial e Integral datadas de 1975, produzidas pelo Professor Aladim Valverde ${ }^{12}$ foram importantes para a definição do Cálculo como uma disciplina acadêmica passível de investigação histórica. Com aproximadamente setenta folhas, versando sobre números reais, limites, continuidades e derivadas tais notas foram utilizadas pelo Professor também em cursos posteriores nos anos 80 do século passado. Para Chervel (1990) o ensino de exposição e os exercícios figuram entre os constituintes de uma disciplina.

Outra fonte importante foi um caderno de Cálculo Diferencial e Integral II do ano de 1976, do aluno Luiz Carlos Pires, que foi ofertado ao Professor Aladim ao final do curso por ele ministrado. Gesto que mostra a importância desse material no percurso acadêmico desse aluno. Essa era uma prática bastante frequente entre os alunos diante de um bom professor, um gesto de gratidão pela aprendizagem possibilitada.

O caderno em questão é de modelo espiral, tamanho pequeno, com aproximadamente oitenta páginas. Confeccionado com extremo capricho pelo aluno Luiz Carlos, que posteriormente veio a se tornar professor de Matemática da rede particular de Juiz de Fora, é constituído de quatro capítulos que foram redigidos no período de 26 de fevereiro a 03 de junho de 1976.

Os cadernos constituem um valioso referencial sobre as práticas docentes de tempos anteriores por possibilitar a representação do passado das ações didático-pedagógicas exercidas em sala de aula. O caderno é um elemento da cultura acadêmica quando se busca conhecer, compreender e analisar o que ocorreu nas instituições de ensino superior no que diz respeito à produção/disseminação de saberes.

A análise dos apontamentos do Professor Aladim confrontada com a do caderno de seu aluno revela uma caprichosa organização e uma acentuada preocupação metodológica por parte deste professor.

Mas em entrevista esse Professor reconhece as dificuldades dos alunos frente à disciplina de Cálculo, traduzidas em reprovação: "Em qualquer tempo sempre teve muita reprovação em Cálculo. Apesar de dar toda a ajuda aos alunos, com trabalhos de verificação, trabalhos em sala e para casa, ainda assim a reprovação era muito grande." (VALVERDE apud RAAD, 2012, p.124).

Aqui a reprovação em Cálculo é tratada como um fato inerente a esta disciplina, constituindo-se como um traço da cultura do seu ensino.

A reprovação em Cálculo persistia em meados dos anos 70 do século passado, mesmo sob a regência de um professor reconhecido por suas qualidades didáticas. A análise das atas departamentais produzidas pelo Departamento de Matemática da Univer-

12. Ex-professor do Departamento de Matemática da UFJF. Lecionou Cálculo entre as décadas de 1970 e 1990. 
sidade Federal de Juiz de Fora durante os anos 70 e 80 do século passado permitiu constatar a preocupação do Departamento com o elevado índice de reprovação em Cálculo.

Foram ofertados cursos intensivos de Cálculo e disponibilizados monitores durante toda a década de 1970. Em 1982, foi criada uma comissão para o estudo da larga reprovação em Cálculo.

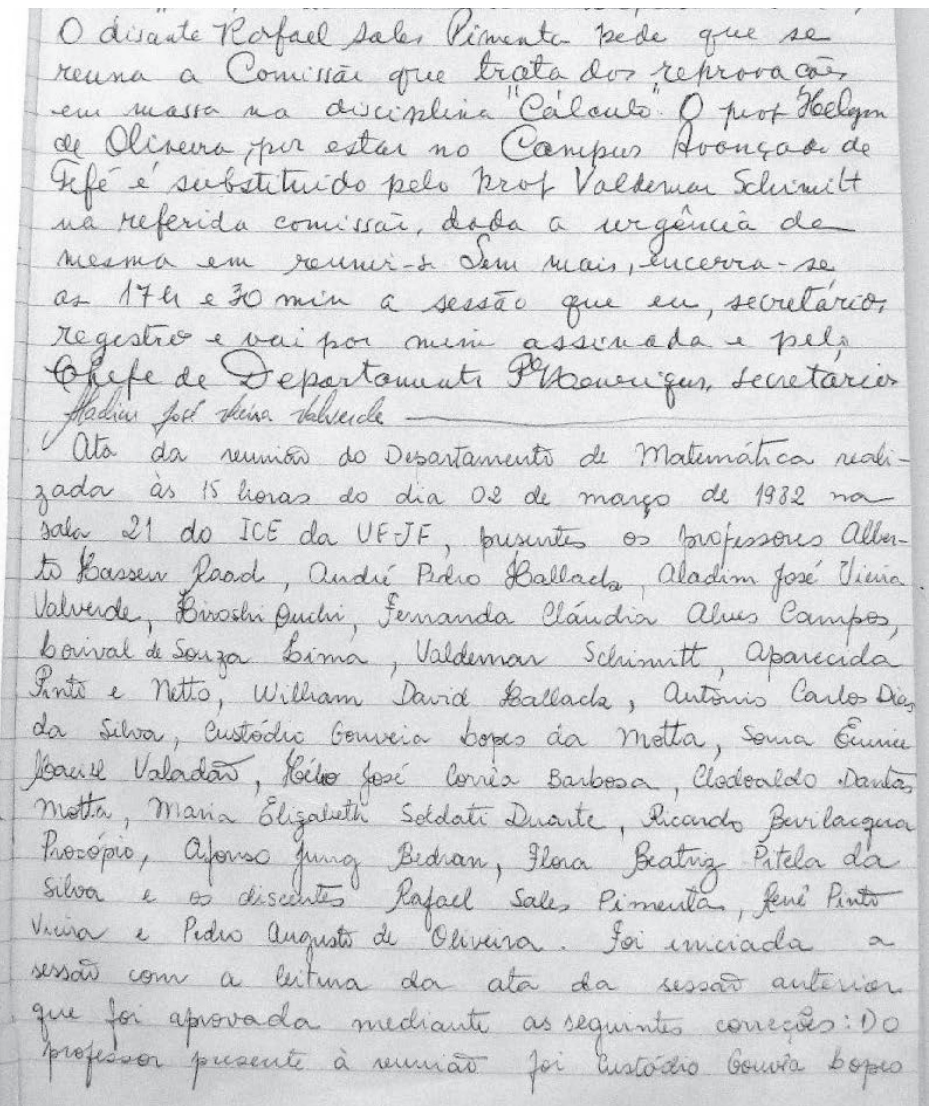

Ilustração 1 Extrato da ata departamental de 12 de janeiro de 1982, que contém a solicitação do discente por reunião da referida comissão.

Outra iniciativa para lidar com a questão da reprovação em Cálculo foi o aumento da carga horária, a partir do primeiro semestre de 1980, quando a disciplina passou de quatro a seis aulas semanais. A ampliação de carga horária parece ter sido um fator criado para melhorar o nível de aprendizagem dos alunos, uma vez que não previa aumento de conteúdo, possibilitando um curso com mais exercícios e revisões. 
Um aspecto identificado pela análise das notas de aula e pelas entrevistas com o Professor Aladim é a relação entre os conteúdos básicos de Matemática, os chamados pré-requisitos, e as dificuldades de aprendizagem dos alunos em Cálculo. Este é um tema recorrente na cultura de ensino desta disciplina (RAAD, 2012).

Intimamente ligada à questão dos pré-requisitos esta a reprovação. Na cultura de ensino de Cálculo em que se privilegia o linear, isto é, um conhecimento só é aprendido bem se o conteúdo que o antecede também for assimilado corretamente, a reprovação é uma consequência natural se esta sequência não for seguida à risca. Essa "dependência" é mais um elemento que naturaliza a reprovação.

Para evitar a reprovação, pela lógica desta cultura do linear, deve-se investir em mais Matemática básica, em cursos chamados por alguns de Matemática Zero ou ainda de Pré-Cálculo.

$\mathrm{Na}$ análise das atas departamentais de 1988, encontra-se um pedido do Coordenador do Curso de Engenharia Civil ao Departamento de Matemática para a introdução de uma disciplina de nivelamento obrigatória aos egressos do vestibular daquele ano com o intuito de subsidiar o Curso de Cálculo no que diz respeito aos pré-requisitos.

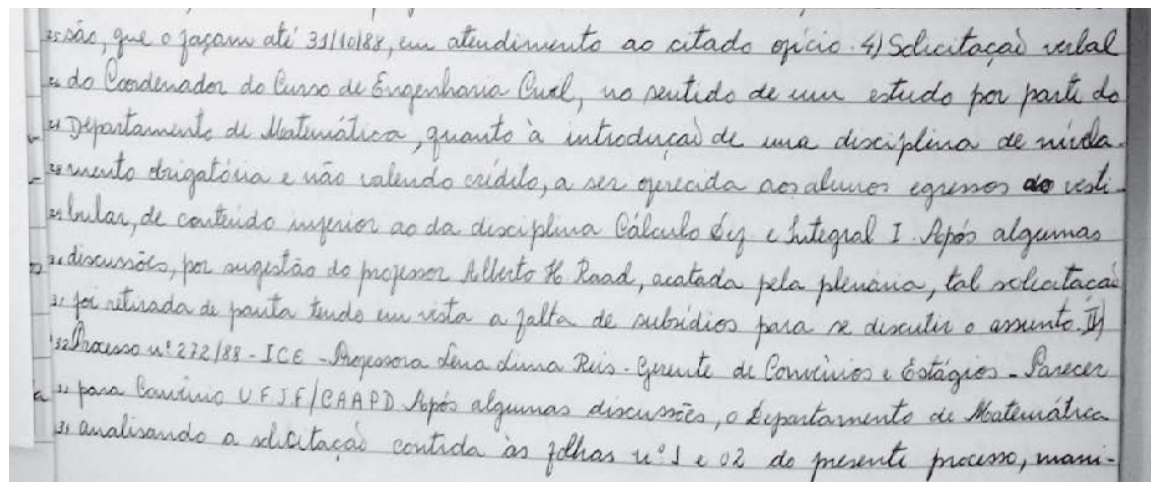

Ilustração 2: Extrato da ata departamental de 17 de outubro de 1988. Solicitação de Matemática Elementar para os estudantes do primeiro período de Engenharia Civil.

As soluções propostas para a problemática dos pré-requisitos sempre defenderam as revisões dos conteúdos e o aumento da carga de Matemática básica. Mas ao que tudo indica tais iniciativas não tem mostrado a eficácia esperada. 


\section{Considerações Finais}

Apesar da existência de bons livros didáticos, de boas práticas pedagógicas, de diferentes iniciativas no sentido de diminuir o insucesso dos estudantes em Cálculo: oferecimento de monitorias, revisão de conteúdos de Matemática básica, diminuição do rigor e valorização de aspectos intuitivos e aplicativos, ainda assim a reprovação persiste, permanece como um problema crônico, uma verdadeira tradição.

Em alguma medida, a relação entre um bom professor/um bom curso de Cálculo se dá na mesma proporção do índice de reprovação dos estudantes. A reprovação ainda está muito mais associada à qualidade do curso do que à inadequação do mesmo ao grupo de estudantes ou ao momento sociocultural. Assim, um curso considerado bom é sinônimo de forte, o que implica em significativo número de reprovados.

Sendo assim, qualquer ação pedagógica que rompa esta tradição da reprovação, passada de década para década, de geração para geração, será vista como uma ameaça à estabilização desta disciplina. Por ser a Educação Matemática uma ciência que se preocupa com a inclusão, o exercício do ofício de historiador desta área pretende por em questão esta cultura de reprovação presente no ensino de Cálculo. E, além disso, enfatizar que medidas consideradas como fundamentais aumento de carga horária, revisões, ampliação do número de exercícios, criação de subdisciplinas como Cálculo Zero - quando colocadas em perspectiva histórica, revelam-se como paliativos com sucesso relativo pontual, não modificando a cultura de reprodução estabelecida.

De fato, sabe-se que mudanças culturais são lentas. Em termos da cultura escolar que envolve a formação de professores de matemática e, mais especificamente, aquela ligada ao ensino de Cálculo - disciplina inauguradora da formação desse profissional em nossas faculdades e universidades - pode-se interrogar como a situação poderia ser mudada. A resposta poderá ser dada, também, na análise em perspectiva histórica: será a transformação do corpo docente do ensino superior, em suas diferentes gerações, que mudará a cultura de reprovação. Em realidade, a incorporação crescente de educadores matemáticos substituindo professores de matemática, poderá alterar a cultura da reprovação em termos da melhor formação do profissional para o ensino na escola básica.

\section{Referências}

ANACLETO, G. M. C. Uma investigação sobre a aprendizagem do Teorema Fundamental do Cálculo. Dissertação (Mestrado), Pontifícia Universidade Católica, São Paulo, 2007. 
BARBOSA, M. A. O Insucesso no ensino e aprendizagem na disciplina Cálculo Diferencial e Integral. Dissertação (Mestrado), Pontifícia Universidade Católica, Curitiba, 2004.

CHARTIER, R. La historia o la lectura del tempo. Madrid: Gedisa, 2007.

CHERVEL, A. A História das disciplinas escolares: reflexões sobre um campo de pesquisa. In: Teoria \& Educação. Porto Alegre: Pannonica, 1990. n.2, p. 117-229.

CURY, H. N. Análise de erros: o que podemos aprender com as respostas dos alunos. Porto Alegre: Autêntica, 2007.

DUARTE, A. R. S. Matemática e educação matemática: a dinâmica de suas relações ao tempo do Movimento da Matemática Moderna no Brasil. São Paulo, 2007. Tese (Doutorado em Educação Matemática) - Pontifícia Universidade Católica.

ESCARLATE, A.C. Uma Investigação Sobre a Aprendizagem da Integral. Dissertação (Mestrado) Universidade Federal do Rio de Janeiro, Rio de Janeiro, 2008.

FRAGOSO, W. C. História da Matemática: uma disciplina do curso de Licenciatura em Matemática da Universidade Federal de Juiz de Fora. 2011. 210 f. Dissertação (Mestrado Profissional em Educação Matemática) - Instituto de Ciências Exatas, Universidade Federal de Juiz de Fora, Minas Gerais, 2011.

OLIVEIRA, M. C. A.; Fragoso, W. C. História da Matemática: história de uma disciplina. Revista Diálogo Educacional (PUCPR. Impresso), v. 11, p. 625/01643, 2011.

OLIVEIRA, M.C. A. A formação matemática de um matemático e educador matemático. In: Wagner Rodrigues Valente. (Org.). Ubiratan D’Ambrósio. $1^{a}$ ed. São Paulo: Annablume, 2007, v.01, p.55-76.

RAAD, M. R. História do ensino de Cálculo Diferencial e Integral: a existência de uma cultura. Dissertação (Mestrado), UFJF, Juiz de Fora, 2011.

SCHUBRING, G. Como Relacionar Histórias Regionais a Padrões Gerais de História? O caso do ensino de matemática na Westphalia. BOLEMA, v. 23, n. 35, p.101-122, 2010. 
SILVA, C. M. S. da. A Faculdade de Filosofia, Ciências e Letras da USP e a formação de professores de matemática. In: REUNIÃO ANUAL DA ANPEd, 23ª Anais... Caxambu: ANPEd, 2000.

SOUZA, V. G. Entrevista concedida a Maria Cristina Araújo de Oliveira, 03 out. 2011, São Paulo.

VALENTE, W. R. História da Educação Matemática: interrogações metodológicas. In: REVEMAT - Revista Eletrônica de Educação Matemática, v.2.2, p. 28-49, UFSC, 2007. Disponível em: <http://www.redemat.mtm.ufsc.br/revemat_2006. htm>. Acesso em: 04 jan.2011, 08h50min.

VALVERDE, A. J. V. Entrevista concedida a Marcos Ribeiro Raad, em 05 de junho de 2011.

VIÑAO FRAGO, A. História das disciplinas escolares. Revista Brasileira de História da Educação. Campinas, SP. SBHE/Editora Autores Associados. Jan./jun, no. 18 Set/dez 2008. 